\title{
Processos expansivos orbitoesfenoidais: estudo anatomopatológico de 82 casos
}

\author{
Orbito-sphenoidal expansive processes: an anatomopathological \\ study of 82 cases
}

\author{
Leonardo Leiria de Moura da Silva ${ }^{1}$ \\ Ligia Maria Barbosa-Coutinho ${ }^{2}$
}

Trabalho desenvolvido no Programa de Pós-graduação em Patologia da Fundação Faculdade Federal de Ciências Médicas de Porto Alegre - Porto Alegre (RS) Brasil.

${ }^{1}$ Estagiário do Serviço de Oftalmologia do Complexo Hospitalar Santa Casa de Porto Alegre (RS). Médico Pesquisador do Programa de Pós-graduação em Patologia da Fundação Faculdade Federal de Ciências Médicas de Porto Alegre - FFFCMPA - Porto Alegre (RS) - Brasil.

${ }^{2}$ Médica Neuropatologista. Livre-docente, Professora Emérita e Titular de Anatomia-patológica, Professora do Programa de Pós-graduação em Patologia da FFFCMPA - Porto Alegre (RS) - Brasil.

Endereço para correspondência: Leonardo Leiria de Moura da Silva. Programa de Pós-graduação em Patologia. Fundação Faculdade Federal de Ciências Médicas de Porto Alegre. Rua Sarmento Leite, 245 - Anexo I

- Porto Alegre (RS) CEP 90050-170

E-mail: leonardolms@gmail.com

Recebido para publicação em 11.01.2007

Última versão recebida em 22.08.2008

Aprovação em 12.11.2008

Nota Editorial: Depois de concluída a análise do artigo sob sigilo editorial e com a anuência do Dr. Eduardo Ferrari Marback sobre a divulgação de seu nome como revisor, agradecemos sua participação neste processo.

\begin{tabular}{|c|}
\hline RESUMO \\
\hline 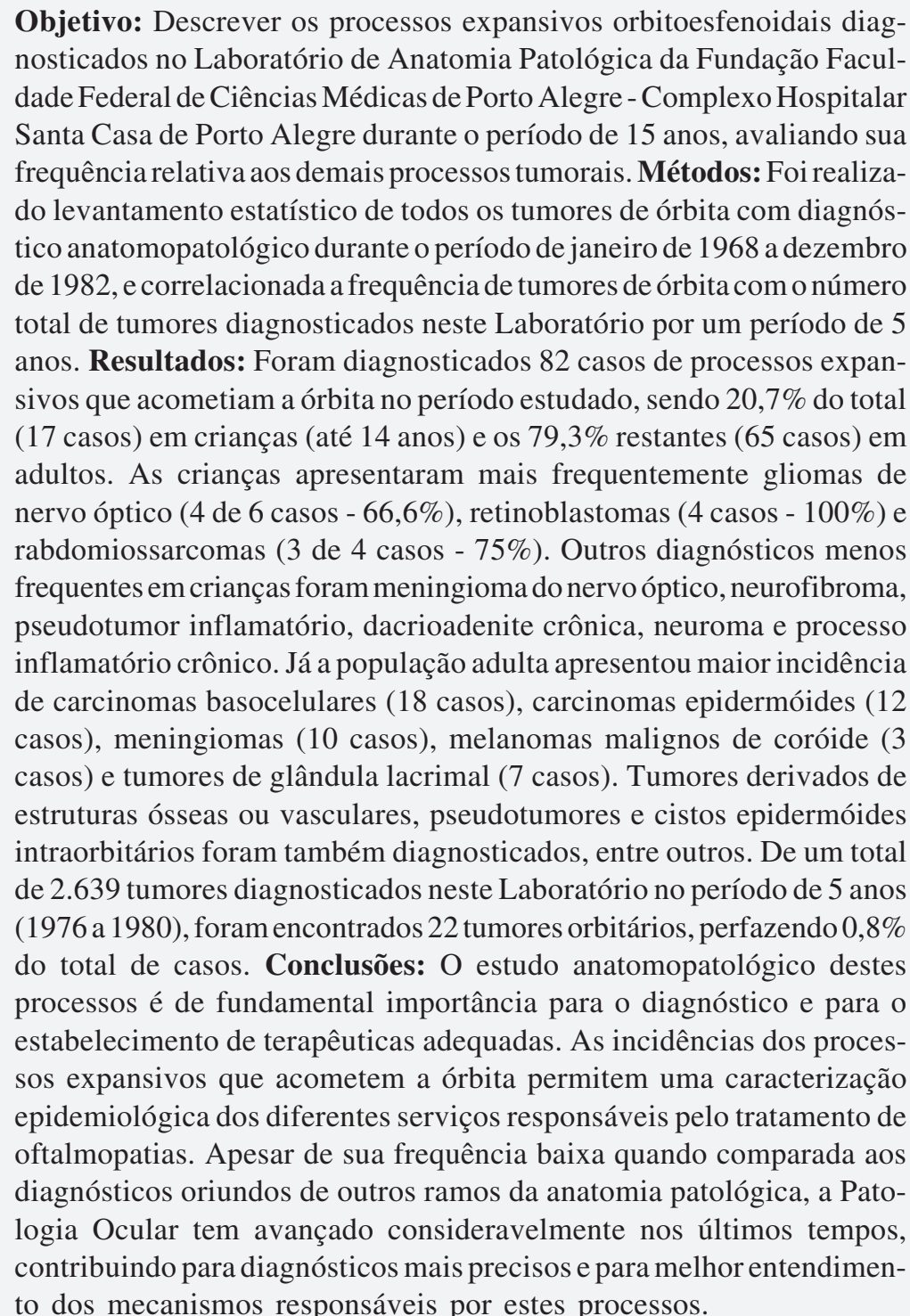 \\
\hline
\end{tabular}

Descritores: Neoplasias oculares; Invasividade neoplásica; Neoplasia orbitárias/patologia; Estudos retrospectivos 


\section{INTRODUÇ̃̃O}

A órbita é uma complexa estrutura, constituída por um arcabouço ósseo revestido pela musculatura extrínseca do globo ocular e por seus tendões, pelo globo ocular com o nervo óptico e pela glândula lacrimal. A patologia expansiva da órbita está representada por tumores que se originam primariamente nas estruturas que a formam e secundariamente a partir de neoplasias que a invadem por via hematogênica ou por continuidade, quer a partir da pele e seios paranasais, como a partir da cavidade intracraniana ${ }^{(1-4)}$. Teoricamente qualquer tipo de tumor de tecidos moles pode afetar a órbita ${ }^{(5)}$.

A incidência dos tumores orbitoesfenoidais varia com a idade, sendo as crianças mais comumente afetadas por retinoblastomas invadindo a órbita, gliomas do nervo óptico, cistos dermóides, angiomas e rabdomiossarcomas ${ }^{(6-7)}$, enquanto que, nos adultos, as neoplasias mais frequentemente encontradas são os carcinomas metastáticos ou que invadem a órbita por continuidade. As metástases orbitárias são mais comuns em tumores primários da mama e da próstata ${ }^{(8)} \mathrm{com}$ notável predominância em pacientes entre 40 a 79 anos de idade. O melanoma de coróide, apesar de infrequente, é o tumor primário intraocular mais comum na população adulta, podendo invadir a órbita por contiguidade e acometendo principalmente pacientes caucasianos na $6^{\underline{a}}$ década de vida ${ }^{(9)}$. Também encontramos nessa faixa etária tumores de glândula lacrimal, meningiomas e os chamados pseudotumores ${ }^{(10-12)}$.

Os tumores orbitários na população adulta são predominantemente malignos, estando mais associados a processos sistêmicos quando comparados aos da população em geral e aos tumores em crianças ${ }^{(4)}$. As incidências destes processos são extremamente variáveis, sofrendo, entre outros, influência do tipo de material estudado, da faixa etária da população acometida, da localização geográfica e do Serviço no qual tiveram sua terapêutica conduzida ${ }^{(3)}$.

$\mathrm{O}$ estudo anatomopatológico dos processos expansivos orbitários tem avançado consideravelmente nas últimas décadas, e a Patologia Ocular tem se estabelecido como um importante ramo da Anatomia Patológica. O desenvolvimento de modelos experimentais nesta área permitiu um maior entendimento dos mecanismos responsáveis pelo estabelecimento dos processos patológicos do olho e de seus anexos, contribuindo sobremaneira para a maior precisão diagnóstica e terapêutica nesses processos.

Este trabalho tem o objetivo de estudar os processos expansivos orbitoesfenoidais diagnosticados em nosso meio, avaliando a sua frequência relativa aos demais processos neoplásicos.

\section{MÉTODOS}

Este estudo se baseia no levantamento estatístico realizado no Laboratório de Anatomia Patológica da Fundação Faculdade Federal de Ciências Médicas de Porto Alegre
(FFFCMPA) - Complexo Hospitalar Santa Casa de Porto Alegre (CHSC) no período de 15 anos entre janeiro de 1968 e dezembro de 1982. Foram analisados todos os tumores de órbita diagnosticados no nosso laboratório no período descrito, e excluídos do presente trabalho aqueles processos expansivos que apresentavam localização exclusiva intraocular e gliomas nascidos em quiasma óptico que não comprometeram a porção intraorbitária do nervo óptico. A frequência do número de casos de tumores de órbita relacionada com o número total de tumores examinados neste Laboratório foi feita por um período de 5 anos (1976 a 1980).

Todos os casos incluídos no presente estudo tiveram seu diagnóstico anatomopatológico confirmado por um dos autores. A partir da pesquisa dos prontuários destes pacientes foram obtidos dados referentes à localização tumoral e à caracterização epidemiológica da amostra. Não foi realizado nenhum tipo de intervenção clínica ou cirúrgica nos pacientes em conseqüência deste estudo, uma vez que este baseou-se somente no levantamento de dados referentes ao diagnóstico anatomopatológico dos espécimes cirúrgicos analisados no laboratório, sendo sempre preservada a confidencialidade dos dados. O presente estudo foi aprovado pelo Comitê de Ética

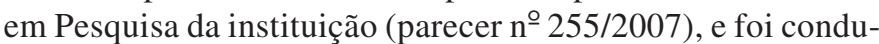
zido dentro dos critérios da resolução 196/1996 do Conselho Nacional de Saúde (CNS).

\section{RESULTADOS}

No período de 15 anos estudado, o total dos processos expansivos comprometendo a órbita diagnosticados no Laboratório de Patologia da FFFCMPA - CHSC foi de 82 casos, com média de idade de 42,4 $\pm 23,9$ anos de idade, oscilando entre 9 meses e 88 anos, distribuídos conforme demonstrado no quadro 1 .

Os processos expansivos orbitoesfenoidais comprometeram crianças (até 14 anos) em 17 casos (20,7\%) e adultos em 65 casos $(79,3 \%)$. Quando comparados à população adulta, as crianças apresentaram mais frequentemente gliomas do nervo óptico (4 de 6 casos - 66,6\%), rabdomiossarcomas (3 de 4 casos - 75\%) e retinoblastomas invadindo a órbita (4 de 4 casos - 100\%). Um dos casos de retinoblastoma apresentou propagação para a região suprasselar, atingindo o espaço subaracnóideo e determinando meningite neoplásica (Figura 1A). Somente um caso de meningioma nascido na membrana aracnóide que reveste o nervo óptico ocorreu em uma criança (1,2\% do total). Outros achados menos frequentes (cada um representando $1,2 \%$ do total), corresponderam neurofibroma ( 1 caso), pseudotumor inflamatório (1 caso), dacrioadenite crônica (1 caso), neuroma ( 1 caso) e processo inflamatório crônico da órbita e do nervo óptico (1 caso). O único caso de pseudotumor foi assim classificado por ser um processo inflamatório que comprometia difusamente a musculatura extrínseca da órbita. A distribuição e a frequência comparativa total dos processos expansivos orbitários somente em crianças são demonstradas no quadro 2 . 
Já na população adulta, houve grande predominância dos carcinomas invadindo a órbita, responsáveis por 30 casos (36,5\% do total de casos). Destes, 18 correspondiam a carcinomas basocelulares (60\% dos carcinomas), sendo este o tipo histológico mais comumente encontrado na órbita em indiví-

Quadro 1. Distribuição dos casos de processos expansivos da região orbitoesfenoidal conforme o diagnóstico

\begin{tabular}{lcc} 
Diagnóstico & $\begin{array}{c}\text { Número } \\
\text { de casos }\end{array}$ & $\begin{array}{c}\text { Média de idade } \\
\text { (variação em anos) }\end{array}$ \\
Carcinoma basocelular & 18 & $63,8(40-82)$ \\
Carcinoma epidermóide & 10 & $55,8(28-73)$ \\
Carcinoma indiferenciado & 2 & $42,0(42)$ \\
Meningiomas & 10 & $49,0(27-80)$ \\
$\quad$ Asa do esfenóide (8) & & \\
Intraósseo (2) & & \\
Meningioma do nervo óptico & 1 & 7,0 \\
Melanoma intraocular & 3 & $48,3(43-55)$ \\
Glioma de nervo óptico & 6 & $11,8(2-24)$ \\
Retinoblastoma & 4 & $2,1(9$ m-3) \\
Rabdomiosarcoma & 4 & $13,7(6-30)$ \\
Neurofibroma & 1 & 8,0 \\
Neuroma & 1 & 4,0 \\
Neurofibrolipoma & 1 & 44,0 \\
Adenoma pleomórfico & 5 & $61,4(41-88)$ \\
de glândula lacrimal & & \\
Carcinoma adenóide cístico & 2 & $54,5(40-69)$ \\
Dacrioadenite & 2 & $17,0(6-28)$ \\
Displasia osteofibrosa & 1 & 50,0 \\
Plasmocitoma & 1 & 50,0 \\
Adamantinoma & 1 & 33,0 \\
Angiomas & 2 & $30,0(20-40)$ \\
Cisto epidermóide & 2 & $20,5(20-21)$ \\
Pseudotumor & 3 & $35,0(7-58)$ \\
Processo inflamatório crônico & 1 & 14,0 \\
Pan-oftalmite supurativa crônica & 1 & 56,0 \\
Total & 82 & \\
\hline
\end{tabular}
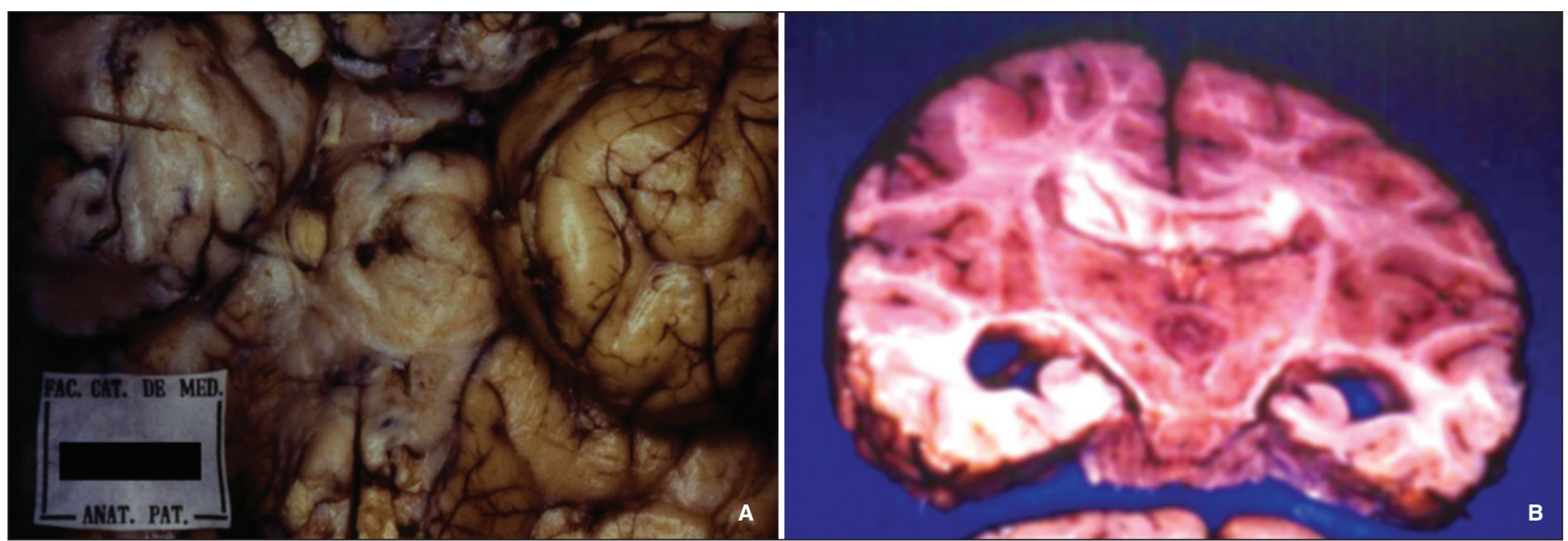

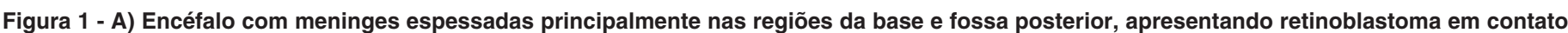

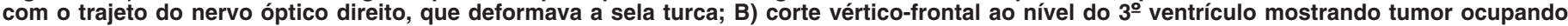
com o trajeto do nervo optico direito, que deformava a sela turca; B) corte vertico-frontal ao nivel do 3 - ventrícu
esta cavidade e infiltrando por continuidade o parênquima cerebral adjacente. duos adultos. Os outros 12 casos de carcinomas que invadiam a órbita corresponderam a carcinomas epidermóides (10 casos - 33,3\% dos carcinomas) e carcinomas indiferenciados (2 casos - $6,7 \%$ dos carcinomas). A maioria dos carcinomas, tanto basocelulares quanto epidermóides, tinha origem na pálpebra. A média de idade dos pacientes com carcinomas orbitários foi de 59,7 $\pm 13,5$ anos, não demonstrando especial predileção por gênero quando comparados (18 homens e 12 mulheres). Os carcinomas basocelulares foram encontrados em igual proporção em homens e mulheres ( 9 casos cada), com média de idade de $63,8 \pm 11,5$ anos, oscilando entre 40 a 82 anos. Já os carcinomas epidermóides, originários principalmente da mucosa olfatória ou dos seios paranasais, foram predominantes em homens (8 de 10 casos), apresentando ligeiro decréscimo da média de idade - 55,8 $\pm 14,6$ anos, variando de 28 a 73 anos quando comparados ao carcinoma basocelular. Os outros dois carcinomas indiferenciados acometeram pacientes do sexo masculino, ambos com 42 anos de idade.

Os meningiomas foram responsáveis por 10 casos em adultos (12,2\% do total). Destes 10 casos de meningiomas, 8 se localizavam junto à asa do esfenóide, sendo dois casos com

\begin{tabular}{|lc|}
\hline $\begin{array}{c}\text { Quadro 2. Distribuição dos processos expansivos orbitoesfenoidais } \\
\text { em crianças }\end{array}$ & Número de casos \\
Diagnóstico & 4 \\
Glioma de nervo óptico & 4 \\
Retinoblastoma & 3 \\
Rabdomiosarcoma & 1 \\
Meningioma de nervo óptico & 1 \\
Neurofibroma & 1 \\
Pseudotumor inflamatório & 1 \\
Dacrioadenite crônica & 1 \\
Neuroma & 1 \\
Processo inflamatório crônico & 17 \\
Total &
\end{tabular}


diagnóstico histológico de meningiomas em placas. Os outros dois meningiomas apresentavam localização exclusivamente intraóssea. Houve predominância do gênero feminino (7 casos) sobre o masculino (3 casos) neste particular tipo de tumor. As idades dos pacientes variaram entre 27 e 80 anos, com média de $49 \pm 15,5$ anos.

Os melanomas oculares, a forma não cutânea mais comum de apresentação desta neoplasia, foram diagnosticados em 3 pacientes $(3,6 \%$ do total). Todos os casos correspondiam a extensões orbitárias de melanomas malignos de coróide. Os pacientes acometidos por esta neoplasia apresentavam média de idade de 48,3 \pm 6,1 anos, oscilando entre 43 e 55 anos.

Os tumores de glândula lacrimal foram encontrados em 7 pacientes adultos (8,5\% do total). O adenoma pleomórfico, tumor derivado do epitélio, que exibe diferenciação tanto epitelial quanto mesenquimatosa, foi o tipo histológico mais comumente encontrado (5 casos). Destes, três apresentavam áreas carcinomatosas associadas a tecido glandular. Em dois casos de tumores de glândula lacrimal (2,4\% do total), o diagnóstico foi de carcinoma adenóide cístico - um tumor altamente infiltrativo, com grande probabilidade de recidiva. Foi diagnosticado somente um caso de dacrioadenite crônica em paciente adulto ( $1,2 \%$ do total).

Os tumores derivados de estruturas ósseas foram infrequentes, representados somente por um caso de displasia osteofibrosa da órbita, um plasmocitoma e um adamantinoma, ( $1,2 \%$ do total - cada caso).

Outras patologias diagnosticadas em menor frequência em adultos foram dois angiomas (2,4\% do total), dois pseudotumores $(2,4 \%$ do total), dois cistos epidermóides intraorbitários (2,4\% do total), uma pan-oftalmite supurativa crônica (processo expansivo tipo abscesso - 1,2\% do total) e um neurofibrolipoma (1,2\% do total).

De um total de 2639 tumores diagnosticados no Laboratório de Anatomia Patológica da FFFCMPA - CHSC no período de 5 anos compreendido entre 1976 e 1980, foram encontrados 22 tumores orbitários, perfazendo $0,8 \%$ do total de casos.

\section{DISCUSS ÃO}

No estudo dos processos expansivos orbitoesfenoidais é passível a ocorrência de diversos fatores que podem influenciar seus resultados finais. Dependendo dos critérios de inclusão de casos, do tipo de diagnóstico ou do serviço no qual estas doenças foram acompanhadas ou tratadas, a proporção destes diagnósticos pode ser extremamente variável. É esperado que casos oriundos de Serviços de Neurocirurgia incluam mais tumores derivados de estruturas intracranianas, como meningiomas e gliomas de nervo óptico ${ }^{(13)}$, por exemplo. Outro fator importante é a idade dos pacientes - Serviços de Oftalmologia Pediátrica tendem a apresentar mais casos de retinoblastomas ou rabdomiossarcomas. Em nosso estudo, acreditamos que, em parte, tais vieses sejam minimizados, pois nele foram avaliados todos os casos de processos expansivos orbitoesfenoidais diagnosticados no nosso Laboratório, independentemente do Serviço que tenha encaminhado material para análise anatomopatológica.

Os processos expansivos orbitoesfenoidais podem manifestar-se clinicamente das mais variadas formas, sendo importante para o médico a diferenciação de casos de proptose secundária a estes processos daqueles casos de pseudoproptose relacionados a outras doenças ${ }^{(3)}$. A causa mais comum de proptose, em adultos, é a doença de Graves, devido a uma fibrose e depósito de mucopolissacarídeos nos músculos extraoculares, que pode se acompanhar de infiltração inflamatória do tipo linfoplasmocitária. Entretanto esse tipo de doença não foi objeto de estudo no presente trabalho, pois nos deteremos a analisar os processos expansivos intraorbitários.

O processo de invasão orbitária nos carcinomas se dá basicamente de duas formas:

- por continuidade, a partir de tumores oriundos ou na mucosa olfatória, ou nos seios paranasais - dos quais o maxilar superior e a conjuntiva são as sedes mais frequentes. O carcinoma basocelular originado nas pálpebras também ocasiona importante comprometimento da órbita por sintopia;

- por via hematogênica, sendo a mama, a próstata e os pulmões os sítios mais comuns de origem das neoplasias primárias.

O carcinoma basocelular é o tumor maligno mais frequente das pálpebras. É um tumor de crescimento lento, que raramente apresenta metástases. Quando acomete a pálpebra, o carcinoma basocelular apresenta comportamento um pouco mais agressivo, devido principalmente à proximidade do globo ocular, e também à grande chance de recidivas pós-cirúrgicas $^{(14)}$. Já o carcinoma epidermóide da pálpebra é um tumor bem menos frequente e que apresenta maiores chances de invasão da órbita, devido ao seu comportamento biológico mais agressivo ${ }^{(15)}$.

Os meningiomas que comprometem a região órbitoesfenoidal podem ser classificados em 4 grupos:

- tumores originados da bainha aracnóide do nervo óptico;

- tumores oriundos do interior da cavidade intracraniana e que se estendem por continuidade para a órbita ou determinam um hiperostose que acaba comprometendo o nervo óptico ou deslocando o globo ocular, podendo ou não comprometer o nervo óptico;

- tumores multicêntricos originados na cavidade intracraniana e no nervo óptico;

- meningiomas extradurais ectópicos, ou seja, intraósseos.

A grande maioria dos meningiomas se situa no grupo daqueles provenientes da cavidade intracraniana e que comprometem a órbita ${ }^{(4)}$. Em nossa estatística encontramos 11 casos de meningiomas que invadiam a órbita, sendo apenas um deles oriundo da bainha meníngea do nervo óptico e outros 3 de origem intraóssea (ectópico). Em 8 casos o tumor teve origem na meninge localizada na asa do esfenóide, posteriormente alcançando e invadindo a órbita. Quando compromete o osso, esse tumor apresenta proliferação meningoteliomatosa no interior dos canais ósseos ou na medula óssea. 
Dos tumores intraoculares que podem invadir a órbita, merecem especial destaque os retinoblastomas e os melanomas. Os retinoblastomas ocorrem mais em crianças, com pico de frequência ao redor dos 2 anos de idade e com prognóstico pior quando do comprometimento do nervo óptico. A incidência de extensão orbitária varia de 7,5 a 10\% dos casos. O diagnóstico precoce e o tratamento cada vez mais conservador são fatores que contribuem significativamente para o aumento da sobrevida e da preservação ocular dos pacientes acometidos por esta neoplasia ${ }^{(16)}$. Pode ocorrer a metastatização de um retinoblastoma para a aracnóide ou para o interior do parênquima nervoso, ou comprometimento por continuidade do nervo óptico. Em nossa casuística de 4 casos desta neoplasia que invadiam a órbita, somente um caso apresentou massa expansiva intracraniana com comprometimento direto do tumor através do nervo óptico (Figuras 1A e 1B), determinando metástase subaracnóidea e meningite neoplásica, achados raros na evolução do retinoblastoma ${ }^{(17)}$. Os demais casos de retinoblastoma apresentavam invasão do nervo óptico. À medida que os retinoblastomas crescem invadindo a órbita, eles ganham acesso a um suprimento vascular mais rico, mudando de padrão para um tumor mais sólido. À microscopia, os retinoblastomas apresentam áreas indiferenciadas, compostas por células redondas, pequenas, com núcleo hipercromático e citoplasma escasso. Podem apresentar as típicas rosetas de Flexner-Wintersteiner, grupos de células cubóides ou colunares curtas dispostas em torno de uma luz central, e as rosetas de Homer-Wright, formadas por arranjos radiais de células em torno de um emaranhado central de fibrilas, conforme é visualizado na figura 2.

Já os melanomas são tumores que podem apresentar comportamento altamente maligno, e quando localizados na parte mais posterior do globo ocular, podem causar compressão do nervo óptico. Ocorrem classicamente mais em adultos, sendo responsáveis por 3 de nossos casos, todos com invasão orbitária por extensão direta (Figuras 3 e 4). A tendência dos melanomas intraoculares é de crescerem para o interior da cavidade vítrea e só tardiamente infiltrarem o nervo óptico

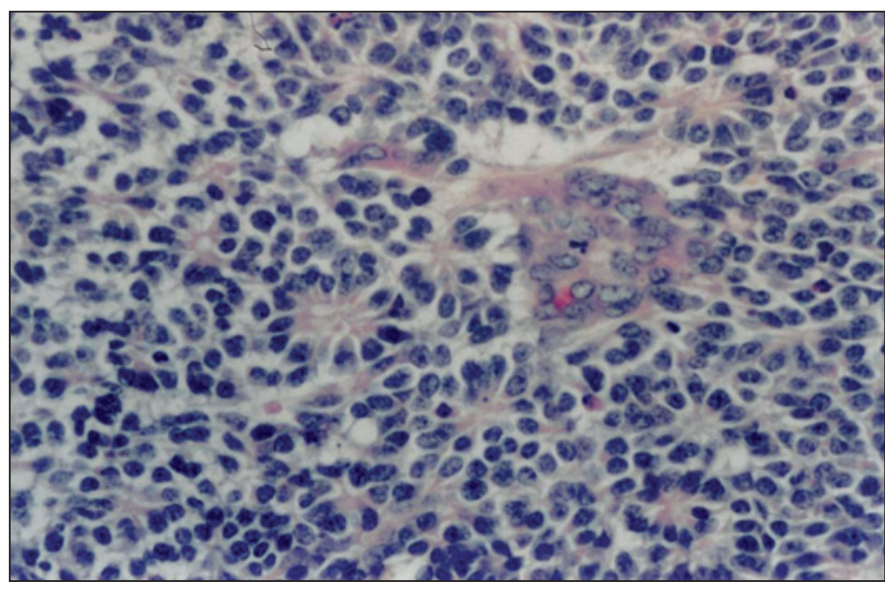

Figura 2 - Aspecto microscópico típico de retinoblastoma, com a presença de rosetas de Homer-Wright e intensa proliferação vascular (HE - 400x) ou as demais estruturas orbitárias ${ }^{(3)}$. A figura 3 mostra o aspecto macroscópico de um melanoma intraocular e a figura 4 demonstra a positividade imuno-histoquímica para o anticorpo HMB-45, altamente específico dos melanomas. Histologicamente, podem ser subdivididos em dois padrões - fusiforme e epitelióide.

Os sarcomas, especialmente os rabdomiossarcomas embrionários, ocorrem mais frequentemente em crianças, e quando localizados na órbita, apresentam melhor prognóstico do que quando localizados em outras áreas ${ }^{(18)}$. Todos os 4 casos estudados ( 3 crianças e 1 adulto) apresentavam localização tumoral retro-orbitária.

A lesão de glândula lacrimal mais frequente em nossa série foi o adenoma pleomórfico (5 casos), um tumor benigno derivado do epitélio, que exibe diferenciação tanto epitelial quanto mesequimatosa (Figura 5). Histologicamente, este tumor apresenta proliferação ductal dentro de um estroma mesenquimal difuso. Destes 5 casos, três apresentavam áreas de degeneração carcinomatosa. A localização habitual destes tu-

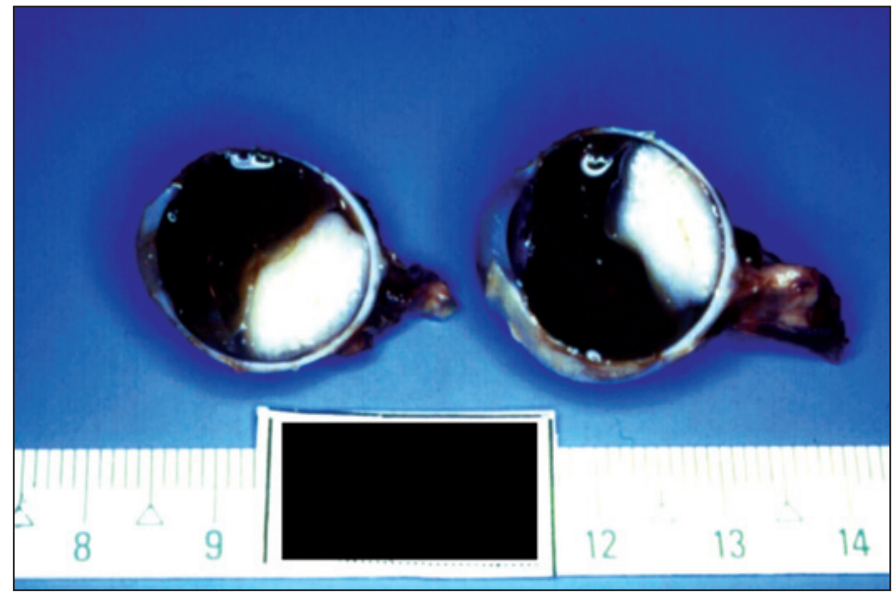

Figura 3 - Corte macroscópico de globo ocular com melanoma intraocular invadindo a órbita

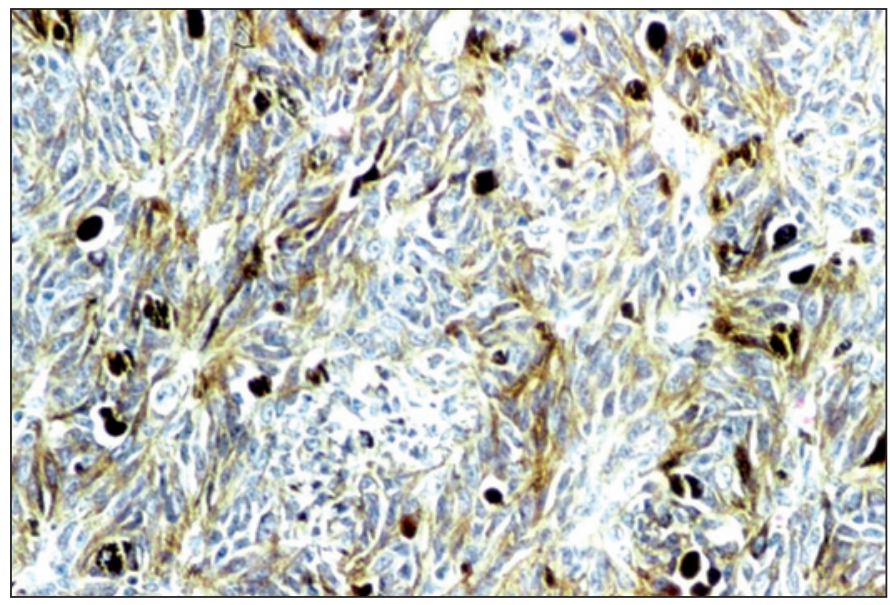

Figura 4 - Melanoma maligno de coróide, evidenciando a presença de células melânicas (HMB45 - 200x) 


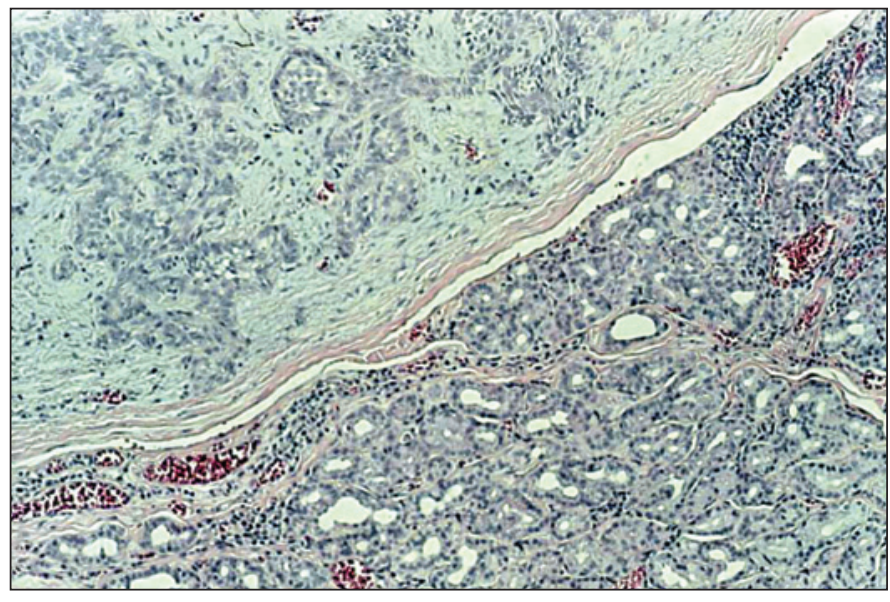

Figura 5 - Aspecto microscópico de um adenoma pleomórfico de glândula lacrimal com limite de glândula normal (à direita) (HE - 100x)

mores é na região orbitária súpero-externa. Dois casos foram diagnosticados como carcinoma adenóide cístico, um tumor com grau maior de anaplasia e mitoses quando comparado ao adenoma pleomórfico ${ }^{(12)}$.

Os tumores derivados de estruturas ósseas são bem menos frequentes. A displasia osteofibrosa é o processo expansivo mais importante, e que pode causar sérias complicações quando a lesão atinge o canal óptico, encarcerando e comprimindo o nervo óptico, que pode se atrofiar gradativamente ${ }^{(12)}$.

O tumor da região orbitoesfenoidal encontrado mais comumente em crianças foi o glioma de nervo óptico (astrocitoma pilocítico), aqui representado por 4 casos, todos eles benignos. Quando confinados ao nervo óptico, os gliomas apresentam prognóstico consideravelmente melhor se comparados aos casos em que o tumor invade o quiasma óptico. Este tipo de tumor apresenta características típicas na tomografia computadorizada e na ressonância magnética, sendo a biópsia diagnóstica raramente necessária nos dias de hoje $\mathrm{e}^{(5,12)}$. Os glioblastomas de nervo óptico são muito raros e não foram vistos no presente estudo.

\section{CONCLUSÃO}

O estudo anatomopatológico dos processos expansivos que acometem a órbita é de fundamental importância para o diagnóstico e para o estabelecimento de terapêuticas adequadas. As incidências destes diferentes processos são dados importantes para o raciocínio e para a investigação diagnóstica das oftalmopatias, e refletem localmente o perfil dos serviços responsáveis pelo manejo destas patologias. A Patologia Ocular é um ramo de contínua expansão dentro da Anatomia patológica, seja através da patologia oftalmológica cirúrgica quanto através da patologia oftalmológica experimental. Novas técnicas diagnósticas, como a técnica de imuno-histoquímica, que permite o diagnóstico histogenético de neoplasias indiferenciadas, e os estudos de imagem, como a tomografia computadorizada e a ressonância magnética, vêm sendo agre- gadas aos métodos clássicos de diagnóstico histológico da Patologia Ocular, contribuindo sobremaneira para a maior precisão diagnóstica e para um melhor entendimento dos mecanismos responsáveis por estes processos.

\section{ABSTRACT}

Purpose: To describe the orbito-sphenoidal expansive processes diagnosed at the Anatomo-Pathological Laboratory of the Fundação Faculdade Federal de Ciências Médicas de Porto Alegre - Complexo Hospitalar Santa Casa de Porto Alegre during a period of 15 years, evaluating their relative frequencies among other tumoral processes. Methods: We performed a statistical analysis of all orbital tumors with anatomicopathological diagnosis from January 1968 to December 1982, comparing the frequency of orbital tumors with the total number of tumors diagnosed at this Laboratory in a period of 5 years. Results: Eighty-two cases were diagnosed of expansive processes involving the orbit in 15 years $-20.7 \%$ of the total (17 cases) affecting children (up to 14 years-old) and the remaining $79.3 \%$ (65 cases) affecting adults. Children presented more frequently optic nerve gliomas (4 of 6 cases $66.6 \%$ ), retinoblastomas (4 cases - 100\%) and rhabdomiosarcomas ( 3 of 4 cases $-75 \%$ ). Less frequent diseases in children were optic nerve meningioma, neurofibroma, inflammatory pseudotumor, chronic dacryoadenitis, neuroma and chronic inflammatory process. The adult population presented more cases of basal cell carcinomas (18 cases), squamous cell carciomas (12 cases), meningiomas (10 cases), choroidal malignant melanoma (3 cases) and lacrimal gland tumors ( 7 cases). Tumors originated from bone or vascular structures, pseudotumors, and intraorbital epidermic cysts were also diagnosed, among others. Of a sum of 2,639 tumors diagnosed at this Laboratory in the time period of 5 years (1976 to 1980), there were 22 cases of orbital tumors, reaching a total of $0.8 \%$ of all cases. Conclusions: The anatomicopathological study of these processes is somehow important to diagnose and to establish an adequate therapy. The incidence of the expansive processes involving the orbit allows an epidemiological characterization of the different medical services responsible for the treatment of eye diseases. Despite its lower frequencies when compared to the diagnosis from other branches of Anatomical Pathology, Eye pathology has developed considerably over the past few years, contributing to more accurate diagnosis and to a better understanding of mechanisms responsible for these processes.

Keywords: Eye neoplasms; Neoplasm invasiveness; Orbital neoplasms/pathology; Retrospective studies

\section{REFERÊNCIAS}

1. Reese AB. Expanding lesions of the orbit. Trans Ophthalmol Soc UK. 1971; 91:85-104. 
2. Jakobiec AF, Zimmermann LE. Symposium on ophthalmic surgical pathology, Part I. Introduction. Hum Pathol. 1982;13(2):98-112.

3. Shields JA. Diagnosis and management of orbital tumors. Philadelphia: W.B. Saunders; 1989

4. Demirci H, Shields CL, Shields JA, Honavar SG, Mercado GJ, Tovilla JC. Orbital tumors in the older adult population. Ophthalmology. 2002;109(2): 243-8. Comment in: Ophthalmology. 2003;110(7):1288; author reply 1288-9.

5. Shields JA, Bakewell B, Augsburger JJ, Flanagan JC. Classification and incidence of space-occupying lesions of the orbit. A survey of 645 biopsies. Arch Ophthalmol. 1984;102(11):1606-11.

6. Shields JA, Bakewell B, Augsburger JJ, Donoso LA, Bernardino V. Spaceoccupying orbital masses in children. A review of 250 consecutive biopsies. Ophthalmology. 1986;93(3):379-84.

7. Kodsi SR, Shetlar DJ, Campbell RJ, Garrity JA, Bartley GB. A review of 340 orbital tumors in children during a 60-year period. Am J Ophthalmol. 1994;117(2): 177-82

8. Shields JA, Shields CL, Brotman HK, Carvalho C, Perez N, Eagle RC Jr. Cancer metastatic to the orbit: the 2000 Robert M Curts Lecture. Ophthal Plast Reconstr Surg. 2001;17(5):346-54

9. Arcieri ES, Fonseca D, França ET, Braga EF, Ferreira MA. [Study of choroidal melanoma at the Federal University of Uberlândia]. Arq Bras Oftalmol. 2002;65(1):89-93. Portuguese.
10. Huh WW, Beverly-Raney R. Orbital metastasis in patients with rhabdomyosarcoma: case series and review of the literature. J Pediatr Hematol Oncol. 2006;28(10):684-7.

11. Seregard S, Sahlin S. Panorama of orbital space-occupying lesions. The 24-year experience of a referral centre. Acta Ophthalmol Scand. 1999;77(1):91-8.

12. Shields JA, Shields CL, Scartozzi R. Survey of 1264 patients with orbital tumors and simulating lesions: The 2002 Montgomery Lecture, Part 1. Ophthalmology. 2004;111(5):997-1008.

13. MacCarty CS, Brown DN. Orbital tumors in children. Clin Neurosurg. 1964; 11:76-93.

14. Ishi LA, Pereira IC, Schellini SA, Marques ME, Padovani CR. Carcinoma basocelular da pálpebra - fatores relacionados com a recidiva tumoral. An Bras Dermatol. 2004;79(4)423-30.

15. Glover AT, Grove AS Jr. Orbital invasion by malignant eyelid tumors. Ophthal Plast Reconstr Surg. 1989;5(1):1-12.

16. Kiratli H, Bilgic S, Ozerdem U. Management of massive orbital involvement of intraocular retinoblastoma. Ophthalmology. 1998;105(2):322-6.

17. Martino ML, Amantea SL, Ulbrich JM, Coutinho LM. Retinoblastoma de extensão supra-selar: registro de um caso. Arq Neuropsiquiatr. 1986;44(2):191-4.

18. Shields CL, Shields JA, Honavar SG, Demirci H. Clinical spectrum of primary ophthalmic rhabdomyosarcoma. Ophthalmology. 2001;108(12): 2284-92. Comment in: Ophthalmology. 2003;110(5):877; author reply 877-8.

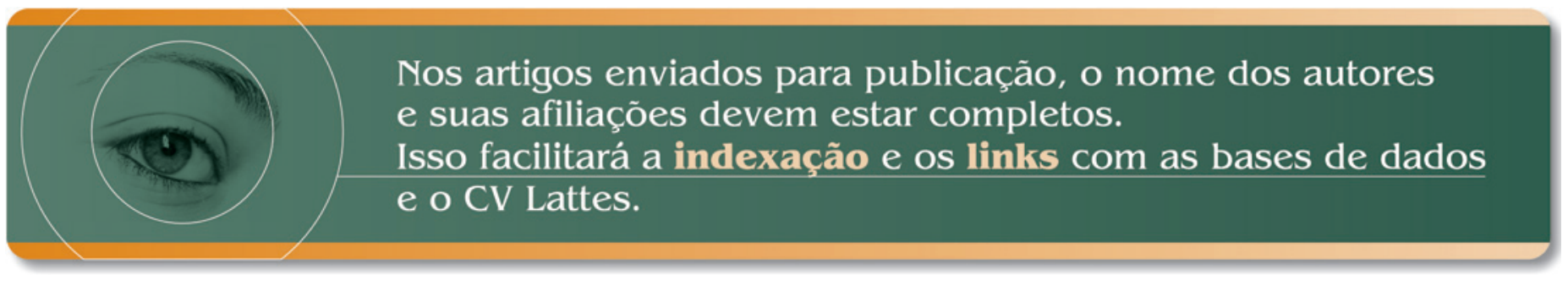

